\title{
IATROGENIC VENTRICULAR SEPTAL DEFECT DURING RIGHT VENTRICULAR ASSIST DEVICE INSERTION IN CONGENITALLY CORRECTED TRANSPOSITION OF THE GREAT ARTERIES
}

\author{
Mimi Deng ${ }^{1}$, Aamir Jeewa ${ }^{1}$, and Osami Honjo ${ }^{1}$ \\ ${ }^{1}$ The Hospital for Sick Children
}

December 23, 2021

\begin{abstract}
This case report describes the management of a large iatrogenic ventricular septal defect (VSD) created by the coring device during systemic ventricular assist device (RVAD) insertion in a 16 year-old patient with congenitally corrected transposition of the great arteries. The VSD was closed by bovine pericardial patch and the ventriculotomy was extended laterally to relocate the VAD sewing ring. After RVAD implantation, patient initially remained cyanotic, potentially due to a tiny VSD patch leak with right to left shunting. Hypoxia was successfully corrected by rescue nitric oxide infusion and patient was bridged to transplant after 91 days.
\end{abstract}

Case Report

\section{IATROGENIC VENTRICULAR SEPTAL DEFECT DURING RIGHT VENTRICULAR AS- SIST DEVICE INSERTION IN CONGENITALLY CORRECTED TRANSPOSITION OF THE GREAT ARTERIES}

Short Title: Iatrogenic VSD during RVAD implantation

Mimi Xiaoming Deng $\mathrm{MD}^{1,2}$, Aamir Jeewa MB BCh${ }^{3}$, Osami Honjo MD, $\mathrm{PhD}^{1,2}$

${ }^{1}$ Division of Cardiovascular Surgery, Labatt Family Heart Centre, The Hospital for Sick Children, Toronto, Canada

${ }^{2}$ Department of Surgery, University of Toronto, Toronto, Canada

${ }^{3}$ Division of Pediatric Cardiology, The Hospital for Sick Children, Toronto, Canada

IRB approval and clinical trial registration are not applicable. No external sources of funding. Case was displayed as a poster presentation for the International Society for Heart and Lung Transplant Annual Meeting 2021, virtual, April 24-28, 2021.

Total word count: $1390 / 1500$

Corresponding Author: Osami Honjo, MD, PhD

Division of Cardiovascular Surgery, Labatt Family Heart Center,

The Hospital for Sick Children 555 University Avenue Toronto, ON, Canada, M5G1X8

Email: osami.honjo@sickkids.ca 
Phone: +1 416-813-6420 Fax: +1 416-813-7984

Key words: Congenital; Pediatric; Surgery; Transplant

Abstract (97/150 words)

This case report describes the management of a large iatrogenic ventricular septal defect (VSD) created by the coring device during systemic ventricular assist device (RVAD) insertion in a 16 year-old patient with congenitally corrected transposition of the great arteries. The VSD was closed by bovine pericardial patch and the ventriculotomy was extended laterally to relocate the VAD sewing ring. After RVAD implantation, patient initially remained cyanotic, potentially due to a tiny VSD patch leak with right to left shunting. Hypoxia was successfully corrected by rescue nitric oxide infusion and patient was bridged to transplant after 91 days.

\author{
Abbreviations \\ ccTGA - congenitally corrected transposition of the great arteries \\ CPB - cardiopulmonary bypass \\ iNO - inhaled nitrous oxide \\ RV - right ventricle
}

RVAD - right ventricular assist device

TEE - transesophageal echocardiogram

VSD - ventricular septal defect

\title{
Introduction
}

Optimal pump positioning is important for adequate ventricular unloading and post-surgical outcomes for patients undergoing ventricular assist device (VAD) implantation. ${ }^{1}$ Furthermore, the geometry of the ventricles and the position of the ventricular septum in patients with congenitally corrected transposition of the great arteries (ccTGA) are quite different from those in a structurally normal heart, posing a unique technical challenge in systemic right ventricular assist device (RVAD) implantation. Herein we present a case of a 16-year-old male with ccTGA, whose systemic RVAD (HeartMate IIIß) or HM3) implantation was complicated with the creation of an iatrogenic ventricular septal defect (VSD), with special focus on surgical and medical managements for severe systemic right ventricle (RV) dysfunction and torrential systemic tricuspid insufficiency. Consent was granted by the subject patient to publish the case report.

\section{Description}

Our patient with ccTGA had previously undergone VSD patch closure and pulmonary valvotomy as an infant and then later palliated with pulmonary artery banding. He subsequently developed worsening heart failure and was listed for heart transplantation due to progressive systemic RV failure. Following ring annuloplasty of the systemic tricuspid valve, we proceeded to systemic RVAD implantation. As per routine, placement of the HM3 inflow cannula to the RV apex was guided by transesophageal echocardiography (TEE) but optimal image acquisition was challenging. The inflow cannula was to be placed anterior and lateral to the true apex of the systemic RV. The ventriculotomy created by the VAD coring device overrode into the ventricular septum and was hidden underneath the inflow cannula ring (Fig 1A). With initiation of RVAD at $4 \mathrm{~L} / \mathrm{min}$, the patient became severely hypoxic with a $\mathrm{PO}_{2}$ of $45-50 \mathrm{mmHg}$ secondary to the large pulmonic to systemic circulation shunt (i.e right-to-left) which was also identified on TEE and epicardial echocardiogram. Cardiopulmonary bypass $(\mathrm{CPB})$ was re-initiated and the inflow cannula was removed, revealing a large iatrogenic VSD at the cannula insertion point (Fig 1A ). The VSD was closed by bovine pericardial patch and the ventriculotomy was extended laterally to relocate the new RVAD sewing ring (Fig 1B ) using 2-0 Tycron sutures placed as an open technique. After CPB was weaned again, the patient initially remained cyanotic and echocardiography imaging identified a tiny residual VSD patch leak with right to left shunting as a potential cause of hypoxia. 
Due to the prolonged duration and complexity of the case, pursuing residual VSD closure was deemed unsafe. A trial of rescue of inhaled nitric oxide (iNO) was initiated which had the dramatic effect of increasing the arterial saturation from the $70 \%$ range to $90 \mathrm{~s}$. The patient was transferred back to the cardiovascular intensive care unit in stable condition and continued to demonstrate oxygen saturations $>90 \%$, even after weaning iNO and extubation from mechanical ventilation. There were no further issues with regards to either VAD support or saturations for our patient after surgery, and the patient was successfully bridged to transplant after 91 days of RVAD support.

\section{Discussion}

We have learned significant lessons from this unique complication. Iatrogenic VSD in the setting of systemic RVAD causes significant hypoxia due to the significant inflow suction by the VAD from the pulmonic ventricle, resulting in a right-to-left shunt. The iatrogenic VSD was effectively treated with VSD patch closure, a similar technique that is employed for a post-infarction VSD. The second significant challenge was persistent right-to-left shunt and desaturation despite having adequate VSD patch and a small patch leak, highlighting the clinical significance of even small shunts after VAD implantation. This is likely due to the combination of high negative pressure from the inflow cannula in the morphologic RV and dysfunctional and distended subpulmonary left ventricle. The mechanism of the remarkable effect of iNO in our case is somewhat unclear, but perhaps explained by reduction of the afterload and subsequent improved ventricular ejection and decompression of the subpulmonary LV.

Determining the favourable positioning of the inflow cannula in ccTGA is a technical challenge. Using routine TEE offers guidance for cannula insertion in most VAD cases, but was not sufficient in preventing iatrogenic injury in this case. The authors would recommend more detailed pre-planning using cross-sectional imaging, such as computed tomography or magnetic resonance imaging, and the addition of epicardial studies for seating the VAD inflow. Although extremely rare, iatrogenic VSDs have been well reported in adults. ${ }^{2,3} \mathrm{Ba}-$ rioliet al describes acute VSD creation during septal myectomy for hypertrophic obstructive cardiomyopathy that was initially repaired intraoperatively with a large bovine pericardial patch, with delayed percutaneous closure of residual VSD using the Amplatzter multi-fenestrated septal occluder. ${ }^{4}$ Successful transcatheter iatrogenic VSD closure after aortic valve replacement has also been well described. ${ }^{5}$ In our case, the acute desaturation from a large iatrogenic VSD was managed intraoperatively with patch repair, RVAD revision, and the addition of supplementary iNO. The possibility of percutaneous intervention for the residual shunt was discussed. However, considering the technical difficulty of device closure near a VAD inflow and the relative improvement of the patient's clinical status with iNO, further procedural intervention was deferred. The presence of the VSD patch and residual shunt did necessitate an earlier listing for heart transplantation postoperatively compared to patients with uncomplicated VAD placement. This case highlights a unique iatrogenic complication during VAD implantation in a patient with complex congenital heart disease, and the management steps that were taken to troubleshoot hemodynamic instability.

\section{Funding Source}

No external sources of funding.

\section{Disclosure Statement}

Authors have no other financial conflict of interest to disclose.

\section{Author's contribution:}

- Mimi Deng: Drafting article

- Aamir Jeewa: Critical revision of article

- Osami Honjo: Critical revision of article, approval of article

\section{References}

1. Imamura T, Adatya S, Chung B, et al. Cannula and Pump Positions Are Associated With Left Ventricular Unloading and Clinical Outcome in Patients With HeartWare Left Ventricular Assist Device. 
J Card Fail. 2018;24(3):159-166. doi:10.1016/j.cardfail

2. Parsons C, Zhao CB, Huang J. Closure of an iatrogenic ventricular septal defect using a hybrid approach and echocardiographic guidance.Ann Card Anaesth . 2020;23(2):212-215. doi:10.4103/aca.ACA_150_18

3. Rashed A, Gombocz K, Fulop J, Alotti N. Iatrogenic ventricular septal defect: A rare complication of surgical reconstruction of mitral paravalvular dehiscence. Int J Surg Case Rep . 2016;27:107-109. doi:10.1016/j.ijscr.2016.08.024

4. Barioli A, Cardaioli F, Pavei A, Tarantini G. Transcatheter closure of complex iatrogenic ventricular septal defect: A case report. Eur Hear J - Case Reports . 2020;4(3):1-5. doi:10.1093/ehjcr/ytaa106

5. Taleyratne JDS, Henderson RA. Transcatheter Closure of Iatrogenic VSDs after Aortic Valve Replacement Surgery: 2 Case Reports and a Literature Review. Tex Heart Inst J. 2016;43(4):329-333. doi:10.14503/THIJ-15-5083

\section{Figure Legends}

Figure 1. ECHO of A) iatrogenic VSD hidden by sewing ring, interventricular septum is outlined by the dotted line.B) RV after VSD patch closure and lateral repositioning of inflow cannula.

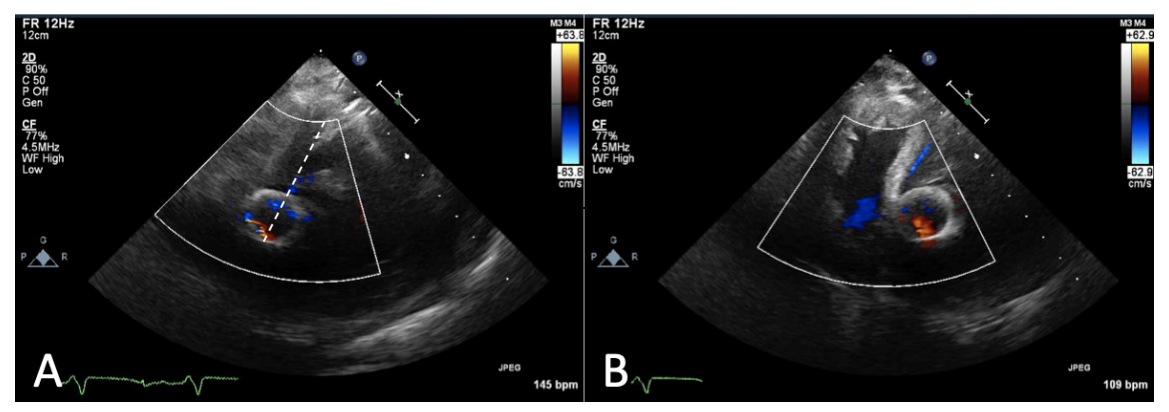

